\title{
Metastatic insulinoma presenting 14 years after benign tumour resection: a rare case and management dilemma
}

\author{
F Keen'1, F Iqbal2, P Owen¹, A Christian³, N Kumar³ and A Kalhan'1 \\ 'Department of Diabetes and Endocrinology, Royal Glamorgan Hospital, Llantrisant, UK, 2Morriston Hospital,
} Swansea, UK, and 3University Hospital of Wales, Cardiff, UK

Correspondence should be addressed to F Keen

Email

fred.keen@btinternet.com

\section{Summary}

We present a 60-year-old woman who underwent successful surgical resection (partial pancreatectomy) for a low grade non-functioning pancreatic neuroendocrine tumour (PNET), with no biochemical or radiological features of recurrence on follow-up visits for 5 years. Fourteen years after the initial surgery, she developed spontaneous severe hypoglycaemic episodes which required hospitalisation, with subsequent investigations confirming the diagnosis of a metastatic insulin-secreting PNET (insulinoma). Medical management of her severe spontaneous hypoglycaemic episodes remained challenging, despite optimum use of diazoxide and somatostatin analogue therapy. Based on a discussion at the regional neuroendocrine tumour multidisciplinary team meeting, she underwent an elective hepatic trans-arterial embolization which was unfortunately unsuccessful. She ended up requiring an emergency right hemihepatectomy and left retroperitoneal mass resection which finally stabilised her clinical condition.

\section{Learning points:}

- Ours is only the seventh case report of a previously benign PNET presenting as a functional insulin secreting metastatic tumour. However, it is the first case report, in which the metastatic functional pNET presented after such a long hiatus (14 years).

- There is currently no clear consensus regarding the length of follow-up of non-functional PNET which are deemed cured post-surgical resection, with most guidelines advocating a median follow up of 5 years (1). The delayed presentation in our case suggests additional considerations should be made regarding optimal post-operative surveillance duration based on the age of the patient, location of the tumour, lymph node spread and Ki-67 index.

- Hepatic artery embolization and/or partial hepatectomy remains a treatment option for PNET patients with significant hepatic metastasis.

\section{Background}

Insulinoma is an extremely rare pNET, with an estimated incidence of two to four cases per 1 million person years (2). About $10 \%$ of these tumours are reported to be functional (malignant) (2), presenting classically with symptoms of hypoglycaemia and weight loss. The diagnosis of an insulinoma has historically been suggested by Whipple's Triad, which consists of hypoglycaemia (blood glucose reading $<3 \mathrm{mmol} / \mathrm{L}$ ), symptoms of hypoglycaemia and relief of symptoms following administration of glucose. However, to diagnose an insulinoma, simultaneous blood tests are needed to show an inappropriately high serum insulin reading $(>10 \mu \mathrm{U} / \mathrm{mL})$ with a low blood glucose $(<3$ $\mathrm{mmol} / \mathrm{L})$, as well as a raised C-peptide level $(>2.5 \mathrm{ng} / \mathrm{mL})$ and a negative sulfonylurea screen. The gold standard for 
diagnosing insulinoma is a $72 \mathrm{~h}$ fast in which to perform these blood tests (3).

The transformation of an established non-functioning pNET into an insulin-secreting metastatic tumour has been reported in only six previous case reports. Ours is the first case of a functional pNET (insulinoma) presenting after such a prolonged hiatus (14 years post what was deemed as a successful resection of a nonfunctional pNET).

\section{Case presentation}

In September 2019, our patient, a 59-year-old woman was found unconscious at home by her family. Her capillary blood glucose levels were noted to be low $(1.1 \mathrm{mmol} / \mathrm{L})$ by the paramedics who, on arrival at the scene, promptly initiated i.v. dextrose infusion as per the protocol. There was a rapid improvement in her cognitive state with this treatment with her subsequent venous blood glucose on arrival to the Accident \& Emergency (A\&E) Department being $8.4 \mathrm{mmol} / \mathrm{L}$. She had experienced coryzal symptoms, arthralgia and episodic confusion for around 2 weeks prior to this admission, but there were no metabolic derangements on her routine biochemical tests. The CT scan of head arranged by the A\&E team also showed a normal appearance.

In 2005, while being investigated for right upper quadrant pain, she was found to have a well-localised cystic tumour, measuring $4 \times 4 \mathrm{~cm}$ in the body of the pancreas on CT. She subsequently underwent a distal pancreatectomy and splenectomy (due to involvement of the splenic artery) along with removal of regional lymph nodes. The histopathology analysis confirmed the diagnosis of a low grade (grade 2), well-differentiated pancreatic neuroendocrine tumour (pNET) with regional lymph node spread (involving two out of the four resected lymph nodes). The resection margins were free from tumour and immunochemistry undertaken had positive staining for chromogranin A, synaptophysin and glucagon with a Ki-67 proliferation index of 5\%. As there were no clinical features suggestive of a functional pNET, with a normal biochemistry profile, immunostaining for insulin or other hormones was not deemed necessary at this stage. She remained under regular clinical surveillance for the next 5 years with no biochemical (fasting gut hormone profile and chromogranin A level) or radiological features (CT thorax, abdomen and pelvis) of disease recurrence or relapse.

Her hospital admission in 2019 was marked by multiple spontaneous hypoglycaemic episodes which were initially treated with infusions of i.v. dextrose, up to $20 \%$ in concentration.

\section{Investigation}

Her initial biochemical and haematological investigations including full blood count, renal and liver function tests, bone profile, inflammatory markers and septic screen, thyroid function test and 09:00 $\mathrm{h}$ cortisol levels were all within the normal ranges. Her venous blood glucose levels, however, were persistently low (range: 1.6 to 13.8 $\mathrm{mmol} / \mathrm{L})$ with an inappropriately elevated C-peptide of $2953 \mathrm{pmol} / \mathrm{L}$ and insulin of $>1000 \mathrm{mU} / \mathrm{L}$ at the time of a venous glucose of $3.3 \mathrm{mmol} / \mathrm{L}$, consistent with an endogenous source of insulin secretion. The sulfonylurea screen was negative. Further blood results later supported this with a chromogranin A of $114 \mathrm{pmol} / \mathrm{L}$ (0-60) and chromogranin B of $226 \mathrm{pmol} / \mathrm{L}$ (0-150) (Table 1).

CT TAP showed multiple large volume peripherally enhancing liver metastases in segments 5, 6, 7 and 8, with the largest deposit lying in the right hepatic lobe measuring $11.3 \mathrm{~cm}$ in diameter (Figs 1 and 2). In addition, there was an $8.2 \mathrm{~cm}$ left para-aortic metastatic tumour deposit (Figs 2 and 3) together with a smaller metastatic deposit anterior to the left renal vein. A subsequent liver biopsy confirmed the diagnosis of a well-differentiated grade 2 NET (Ki67 3\%, synaptophysin and chromogranin positive) (Figs 4, 5, 6, 7 and 8).

\section{Treatment}

Our patient continued to experience severe spontaneous hypoglycaemic episodes despite multiple continuous i.v. dextrose infusions, in addition to a carbohydrate rich diet. She was initiated on a gradually titrating dose of diazoxide, starting at $125 \mathrm{mg}$ three times a day (TDS). This was increased to $150 \mathrm{mg}$ TDS after 9 days, then to $175 \mathrm{mg}$ TDS another 3 days later. Initially, on the lower doses of diazoxide, there was no significant change to her hypoglycaemic pattern. Higher doses of diazoxide,

Table 1 Showing relevant biochemical parameters on admission.

\begin{tabular}{llccc}
\hline & & Value & & Normal value \\
\cline { 1 - 1 } Glucose, $\mathrm{mmol} / \mathrm{L}$ & & 3.3 & & $4-7$ \\
C-peptide, pmol/L & & 2953 & & $<200$ \\
Insulin, $\mathrm{mU} / \mathrm{L}$ & $>1000$ & $<25$ (fasting) \\
Chromogranin A, pmol/L & & 114 & & $0-60$ \\
Chromogranin B, pmol/L & 226 & $0-150$ \\
\hline
\end{tabular}




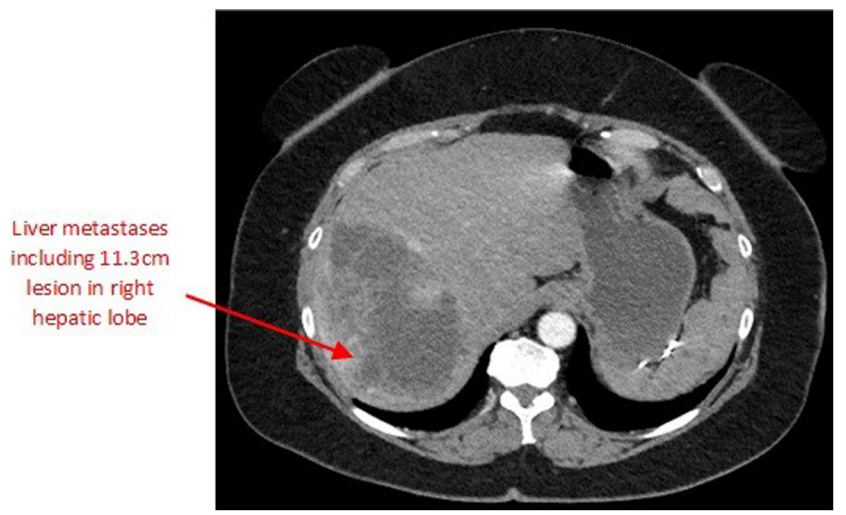

\section{Figure 1}

Contrast-enhanced CT showing liver metastases including a right hepatic lobe lesion measuring $11.3 \mathrm{~cm}$ in diameter.

however, did decrease the frequency of hypoglycaemic episodes, allowing the i.v. dextrose infusions to be stopped, but, unfortunately, she developed side effects of intense nausea, fluid retention (requiring concomitant use of furosemide) and diarrhoea. She also developed a rash which was later biopsied and confirmed to be secondary to a drug reaction. Considering the chronology of clinical events, diazoxide was deemed as the most likely medication which induced this cutaneous reaction. In an attempt to medically manage her hypoglycaemic episodes pending possible surgical intervention, she was initiated on octreotide subcutaneous (SC) injections, starting at $50 \mu \mathrm{g}$ twice daily (BD), increasing up to $200 \mu \mathrm{g}$ TDS over 6 days. This was used alongside a lower dose of diazoxide (100 mg TDS), all the while closely monitoring her plasma glucose readings. However, unfortunately, this combination failed to reduce the frequency or intensity of her severe hypoglycaemic episodes.

Following discussions at the regional neuroendocrine tumour multidisciplinary team meeting (NET MDT), our patient underwent an elective hepatic artery embolization of the culprit liver lesions in an attempt to alleviate her symptoms. It was felt that this would be

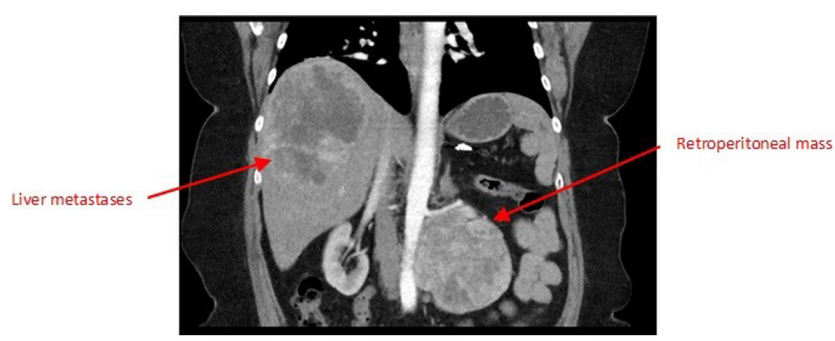

Figure 2

Contrast-enhanced CT showing liver metastases and retroperitoneal mass.

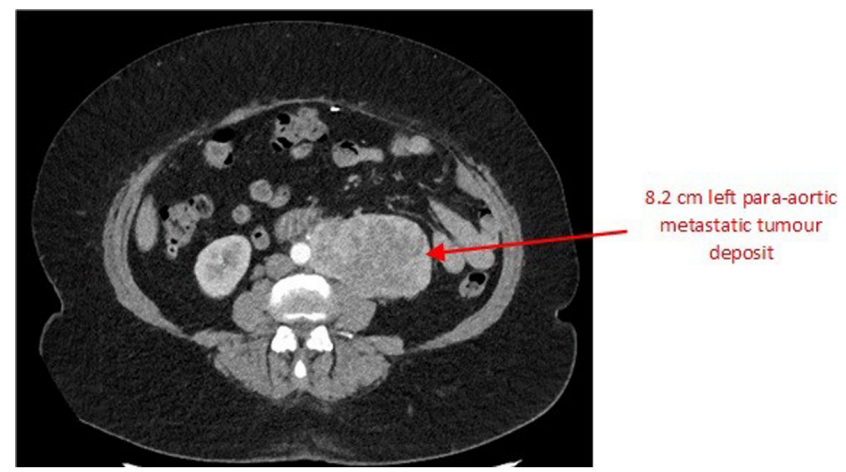

\section{Figure 3}

Contrast-enhanced CT showing liver metastases including a $8.2 \mathrm{~cm}$ left para-aortic metastatic tumour deposit.

successful in stabilising the patient's blood sugars. This was achieved, but lasted only $48 \mathrm{~h}$ before the patient's blood glucose dropped to $2.7 \mathrm{mmol} / \mathrm{L}$, without IV dextrose infusion. Due to the patient's deterioration and the side effects experienced secondary to diazoxide, it was agreed that medical management was not possible and the only option likely to be successful was surgery. An emergency laparotomy was therefore performed to debulk the disease and all macroscopic metastasis was completely resected with a right hemihepatectomy and left retroperitoneal mass resection. She recovered well, with no post-operative complications, and achieved normoglycaemia before being subsequently discharged home.

\section{Outcome and follow-up}

Histology results of the resected tumour deposits were consistent with the original results of a grade 2 welldifferentiated metastatic NET with negative tumour

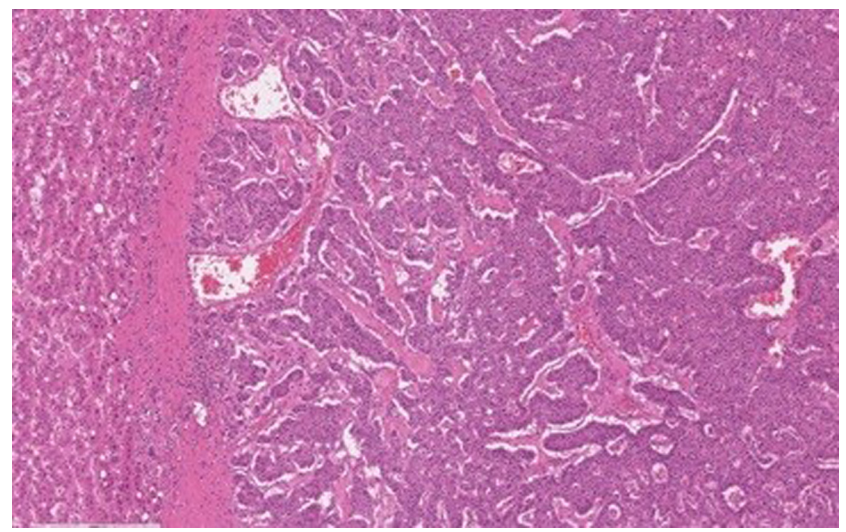

Figure 4

Liver metastasis histology showing a nested and trabecular growth pattern (haematoxylin and eosin staining). 


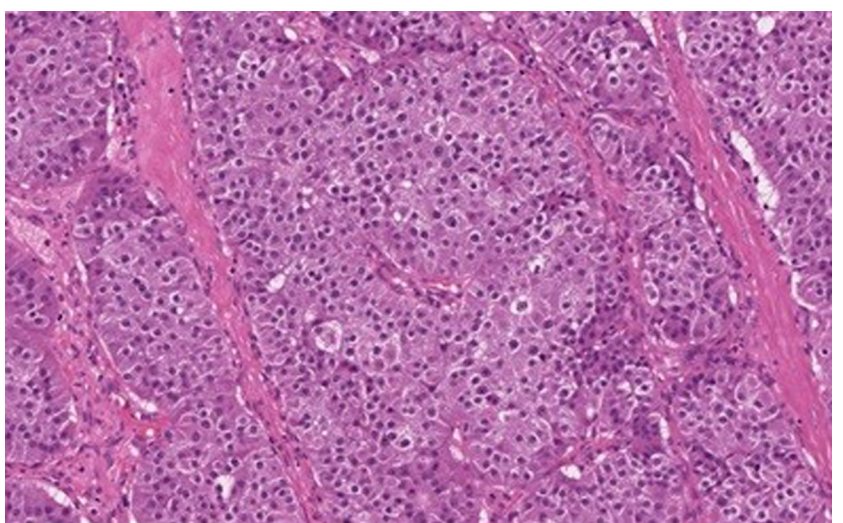

Figure 5

Retroperitoneal mass histology (haematoxylin and eosin staining).

margins. Since the emergency surgery in November 2019, our patient has been clinically stable and remains under biochemical and radiological life-long surveillance.

\section{Discussion}

Pancreatic neuroendocrine tumours (pNET) are uncommon, comprising less than $5 \%$ of all pancreatic tumours and $7 \%$ of all neuroendocrine tumours (4). Eighty percent of these are benign (non-functional), although their incidence is increasing due to an improvement in imaging modalities. Ten percent are part of multiple tumours (such as in multiple endocrine neoplasia type 1 (MEN-1)) and only $10 \%$ are metastatic malignant insulinoma (2). A significant proportion of non-functional pNET may be detected incidentally, as was the case in our patient.

pNET are believed to be derived from the ductal epithelial stem cells (5). Most of these tumours are solitary, sporadic, well differentiated and follow an indolent course,

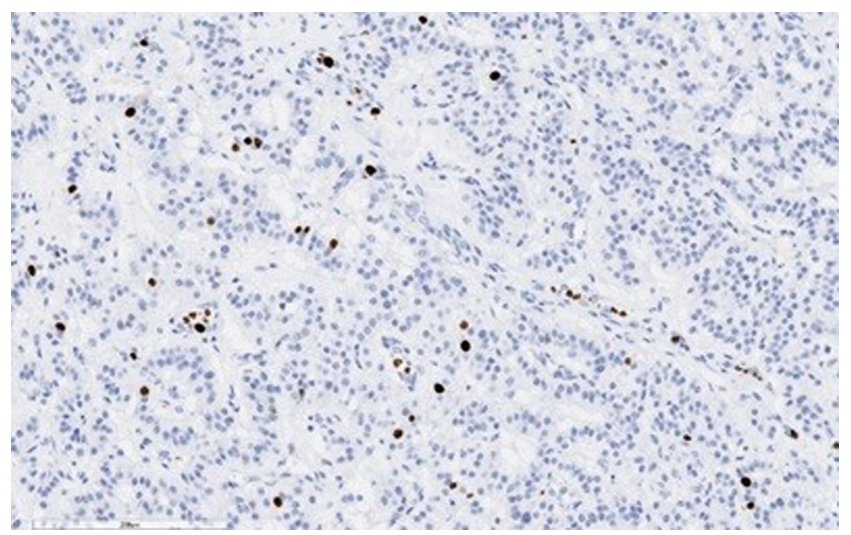

Figure 6

Ki67 stained nuclei.

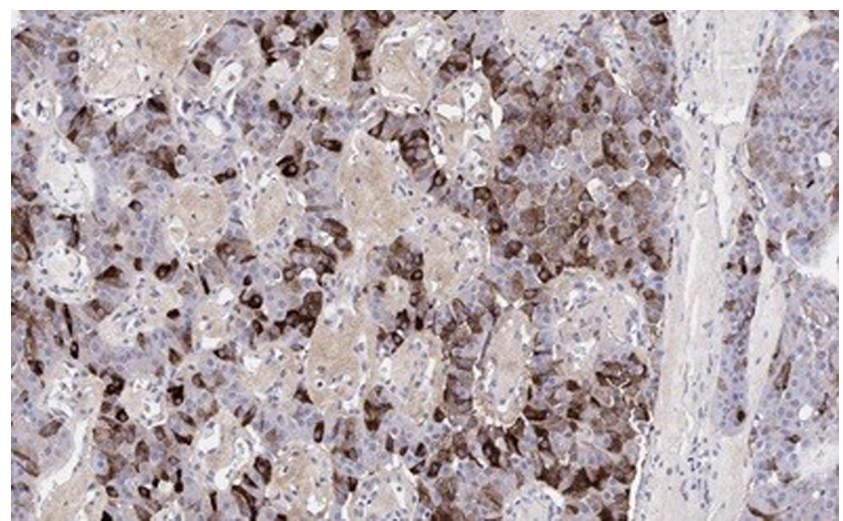

Figure 7

Positive insulin immunostaining in tumour cells.

although familial conditions such as MEN-1, von HippelLindau syndrome (vHL), neurofibromatosis type 1 (NF1) and tuberous sclerosis may be associated with multifocal pNET. Reassuringly, our patient did not have a family history or any other clinical/biochemical characteristics of any of these genetic conditions.

The transformation of an established non-functioning pNET into an insulin-secreting metastatic tumour has only been reported in six previous case reports. However, in most of these cases, patients had not undergone surgical removal of their pNET and displayed malignant clinical characteristics within a relatively short frame of time.

Two cases have been described by Yu et al. (6), one of which included a patient with grade 2 pNET with lymph node metastases who underwent pancreaticoduodenectomy and received adjuvant chemotherapy, but represented 2 years later with hepatic metastases. She finally showed symptoms of hypoglycaemia 7 years after her original diagnosis, although she received continuous

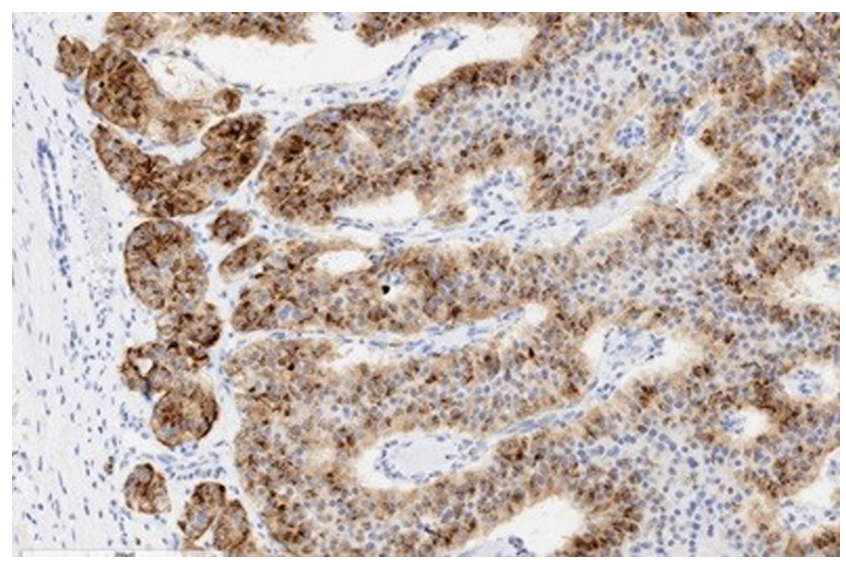

Figure 8

Strongly positive chromogranin A immunostaining. 
treatment (chemotherapy) over this time-frame. The other case described by Yu et al. was of a patient with a grade 1 pNET who had multiple surgical procedures to remove the tumour and eventually presented 5 years after his initial diagnosis with hypoglycaemia secondary to a functional pNET (insulinoma).

Koshy et al. (7) have reported a similar case regarding a woman who presented with recurrent hypoglycaemia 6 years after resection of a non-functioning pNET. However, again, this patient was found to have hepatic metastases 2 years after her original resection and the hepatic lesions were biopsied but not removed. In contrast to the aforementioned case reports, our patient remained asymptomatic and showed no biochemical and radiological features of disease recurrence or relapse for 5 years post-surgical resection of her non-functional pNET.

In three of the other four case reports, at least one liver metastasis was evident at the time of initial presentation $(8,9,10)$. The 4 th report, Arslan et al. (11), documented the case of a 62-year-old male who had initially presented with abdominal pain and was found to have a $20 \times 12 \mathrm{~mm}$ pancreatic tail lesion on CT abdomen. The patient did not agree to the recommended investigations initially as his abdominal pain resolved. Unfortunately, after 24 months, he experienced severe, life-threatening spontaneous hypoglycaemic episodes which were subsequently confirmed to be due to a malignant insulinoma with metastases to the liver and peripancreatic lymph nodes. None of the four previously reported patients were treated with surgical resection, with chemotherapy or immunotherapy being the preferred treatment modalities. The symptoms related to spontaneous hypoglycaemia secondary to functional insulin-secreting pNET all developed within 4 years of initial presentation.

The small number of similar case reports once again highlights the rarity of metastatic insulinoma in clinical practice. From a review of the literature, our patient appears to be particularly unique in having such a delayed presentation of functioning insulinoma after the initial tumour resection.

The onset of severe hypoglycaemic symptoms in our patient was relatively sudden and rapidly progressive, warranting hospital admission. Although the initial surgical resection was complete with clear margins, it is possible that microscopic tumour cells remained dormant in the non-resected pancreas. The relatively low proliferative index of the primary tumour could explain the delayed secondary presentation 14 years later. In addition, the functional transformation (non-functional to insulin secreting) is in line with the previous case reports
$(6,7,8,9,10,11)$, albeit remaining a rare phenomenon. Alternatively, the development of a metastatic insulinsecreting pNET after a previous successful resection of a non-functional pNET could have been a coincidence, with genetic instability contributing to it. This could be a possibility in our patient, given the long interval between the two presentations, albeit less likely than the former theory. The exact molecular mechanism of development of sporadic pNET remains unclear, although, based on SNP studies, four chromosomal abnormalities have been linked to the development of $60 \%$ of them (12). Loss of chromosomes 3q, 6pq, and 10pq, and gains of 5q, 12a, $18 \mathrm{q}$, and $20 \mathrm{q}$ have all been linked with the development of malignant transformations of pNET.

There are no clear guidelines for the median follow-up duration of patients with benign pNET who are deemed cured post-surgical resection. The European Neuroendocrine Tumour Society (ENETS), in their latest guidelines, has made no specific recommendation for the duration of follow up for these patients who undergo successful surgical resection (13). On the other hand, the French NET society guidelines suggest no long-term follow-up is required in the subgroup of patients deemed cured of disease (14). ENETS, however, do recommend 5-year follow-up for high grade gastroenteropancreatic neuroendocrine tumours (1). Consensus guidelines from the Commonwealth Neuroendocrine Tumour Collaboration (CommNETS) in conjunction with the North American Neuroendocrine Tumour Society (NANETS) advocate a follow-up period of 10 years, with particular consideration for more frequent or longer follow-up of patients with a Ki-67 index over 5\% or any number of positive lymph nodes (15). Our patient would therefore fall into this category. Due to the rarity of these tumours and the variation in each case, it seems necessary to take each decision on follow-up on an individualised basis. Metastatic insulin-secreting pNET remain rare and based on the experience of our case, a longer duration of surveillance might be more appropriate in patients presenting at a younger age, with consideration given to Ki-67 index and loco-regional tumour spread.

Our patient underwent a partial pancreatectomy for a pancreatic lesion which was subsequently confirmed to be a low grade, well-differentiated pNET on histology. The patient underwent clinical, biochemical and radiological surveillance for 5 years post-surgery. She presented another 14 years post-elective partial pancreatectomy with spontaneous hypoglycaemic episodes; subsequent investigations confirming diagnosis of a metastatic insulin-secreting pNET. The delayed presentation, in 
this case, highlights the importance of a prolonged or possibly life-long surveillance for such patients, in view of the potential of recurrence of this slow-growing tumour which may remain indolent in body.

\section{Declaration of interest}

The authors declare that there is no conflict of interest that could be perceived as prejudicing the impartiality of the research reported.

\section{Funding}

This research did not receive any specific grant from any funding agency in the public, commercial or not-for-profit sector

\section{Patient consent}

Written informed consent was obtained from the patient for publication of this article and accompanying images.

\section{Author contribution statement}

Dr Frederick Keen wrote the case report. Professor Atul Kalhan, Dr Penelope Owen and Dr Fizzah Iqbal reviewed the case report and managed the medical care of the patient. Mr Nagappan Kumar reviewed the case report and managed the surgical treatment of the patient, both at the initial presentation and 14 years later. Dr Adam Christian was the pathologist involved in care and provided the histology images.

\section{Acknowledgement}

The authors would also like to acknowledge the guidance of Professor Aled Rees in the medical management of the patient.

\section{References}

1 Garcia-Carbonero R, Sorbye H, Baudin E, Raymond E, Wiedenmann B, Niederle B, Sedlackova E, Toumpanakis C, Anlauf M, Cwikla JB, et al. Enets consensus guidelines for high-grade gastroenteropancreatic neuroendocrine tumors and neuroendocrine carcinomas. Neuroendocrinology 2016103 186-194. (https://doi. org/10.1159/000443172)

2 Service FJ, McMahon MM, O’Brien PC \& Ballard DJ. Functioning insulinoma - incidence, recurrence, and long-term survival of patients: a 60-year study. Mayo Clinic Proceedings 199166 711-719. (https://doi.org/10.1016/s0025-6196(12)62083-7)

3 Oberg K, Couvelard A, Delle Fave G, Gross D, Grossman A, Jensen RT, Pape UF, Perren A, Rindi G, Ruszniewski P, et al. ENETS consensus guidelines for the standards of care in neuroendocrine tumors: biochemical markers. Neuroendocrinology 2017105 201-211. (https:// doi.org/10.1159/000472254)
4 Bartolini I, Bencini L, Risaliti M, Ringressi MN, Moraldi L \& Taddei A. Current management of pancreatic neuroendocrine tumors: from demolitive surgery to observation. Gastroenterology Research and Practice 20182018 9647247. (https://doi.org/10.1155/2018/9647247)

5 Lodish MB, Powell AC, Abu-Asab M, Cochran C, Lenz P, Libutti SK, Pingpank JF, Tsokos M \& Gorden P. Insulinoma and gastrinoma syndromes from a single intrapancreatic neuroendocrine tumor. Journal of Clinical Endocrinology and Metabolism 200893 1123-1128. (https://doi.org/10.1210/jc.2007-2449)

6 Yu R, Nissen NN, Hendifar A, Tang L, Song YL, Chen YJ \& Fan X. A clinicopathological study of malignant insulinoma in a contemporary series. Pancreas 201746 48-56. (https://doi. org/10.1097/MPA.0000000000000718)

7 Koshy AA, Gordon IO, Ha TGV, Kaplan EL \& Philipson LH. Metastatic insulinoma following resection of nonsecreting pancreatic islet cell tumor: a case report and review of the literature. Journal of Investigative Medicine High Impact Case Reports 20131 2324709612473274. (https://doi.org/10.1177/2324709612473274)

8 Nahmias A, Grozinsky-Glasberg S, Salmon A \& Gross DJ. Pancreatic neuroendocrine tumors with transformation to insulinoma: an unusual presentation of a rare disease. Endocrinology, Diabetes and Metabolism Case Reports 20152015 150032. (https://doi.org/10.1530/ EDM-15-0032)

9 Vashi PG, Gupta D \& Dahlk S. A unique case of a nonfunctional metastatic pancreatic neuroendocrine tumor transforming Into an insulin-secreting tumor with an unusual clinical course. Pancreas 201140 781-784. (https://doi.org/10.1097/MPA.0b013e318212c42d)

10 Ohn JH, Kim YG, Lee SH \& Jung HS. Transformation of nonfunctioning pancreatic neuroendocrine carcinoma cells into insulin producing cells after treatment with sunitinib. Endocrinology and Metabolism 201328 149-152. (https://doi.org/10.3803/ EnM.2013.28.2.149)

11 Arslan MS, Ozbek M, Karakose M, Tutal E, Ucan B, Yilmazer D, Dilli A, Gultekin SS, Cakal E \& Delibasi T. Transformation of nonfunctioning pancreatic tumor into malignant insulinoma after 3 years: an uncommon clinical course of insulinoma. Archives of Endocrinology and Metabolism 201559 270-272. (https://doi. org/10.1590/2359-3997000000049)

12 Oberg K. Genetics and molecular pathology of neuroendocrine gastrointestinal and pancreatic tumors (gastroenteropancreatic neuroendocrine tumors). Current Opinion in Endocrinology, Diabetes, and Obesity 200916 72-78. (https://doi.org/10.1097/ med.0b013e328320d845)

13 Falconi M, Eriksson B, Kaltsas G, Bartsch DK, Capdevila J, Caplin M, Kos-Kudla B, Kwekkeboom D, Rindi G, Klöppel G, et al. Consensus guidelines update for the management of functional p-NETs (F-pNETs) and non-functional p-NETs (NF-p-NETs). Neuroendocrinology 2016103 153-171. (https://doi.org/10.1159/000443171)

14 Baudin E, Caron P, Lombard-Bohas C, Tabarin A, Mitry E, Reznick Y, Taieb D, Pattou F, Goudet P, Vezzosi D, et al. Malignant insulinoma: recommendations for characterisation and treatment. Annales d'Endocrinologie 201374 523-533. (https://doi.org/10.1016/j. ando.2013.07.001)

15 Singh S, Moody L, Chan DL, Metz DC, Strosberg J, Asmis T, Bailey DL, Bergsland E, Brendtro K, Carroll R, et al. Follow-up recommendations for completely resected gastroenteropancreatic neuroendocrine tumors. Journal of the American Medical Association of Oncology 20184 1597-1604. (https://doi.org/10.1001/jamaoncol.2018.2428) 